\title{
Práticas seguras do/no cuidado de enfermagem no contexto hospitalar: é preciso pensar sobre isso e aquilo
}

Mara Ambrosina de Oliveira Vargas ${ }^{1}$ Anna Maria Hecker Luz ${ }^{2}$

\begin{abstract}
O ensaio reflexivo aborda as práticas seguras, do/no cuidado de enfermagem, no contexto hospitalar. Para tanto, analisa como o cuidar na enfermagem é ressignificado nas diferentes propositivas técnicas e políticas para a almejada ampliação das práticas seguras. Finaliza evocando a necessidade da enfermagem de se capacitar cientificamente e de comprometer-se eticamente com ações sistêmicas de avaliação e prevenção, tentando viabilizar a redução de desfechos indesejados e analisar, criteriosamente, o impacto sobre a qualidade do cuidado.

Descritores: Gerenciamento de segurança; Certificação; Acreditação; Indicadores de qualidade em assistência à saúde; Contenção de riscos biológicos.
\end{abstract}

\begin{abstract}
Safe practices of and in the nursing care within the hospital context: one needs to think on this and that
This reflective essay approaches safe practices of and in the nursing care within the hospital context. For such purpose, it analyzes how the nursing care receives a new meaning among the different technical and political proposals for the expected broadening of safe practices. It ends up by evoking the nursing need of qualifying itself scientifically and of committing itself ethically with systemic actions of evaluation and prevention in the attempt of making feasible the reduction of undesirable outcomes and of analyzing with criteria the impact on the care quality. Descriptors: Safety management; Certification; Accreditation; Quality indicators health care; Containment of biohazards.
\end{abstract}

\begin{abstract}
Prácticas seguras de la y en la atención de enfermería en el contexto hospitalario: hay que pensar acerca de eso y aquello
El ensayo reflexivo aborda las prácticas seguras de la y en la atención de enfermería en el contexto hospitalario. Para tanto, analiza como el cuidar en la enfermería recibe un nuevo significado en las diferentes propuestas técnicas y políticas para la deseada ampliación de las prácticas seguras. Finaliza evocando la necesidad de la enfermería de capacitarse científicamente y de comprometerse éticamente con acciones sistémicas de evaluación y prevención, intentando viabilizar la reducción de resultados no deseados y analizar con criterios el impacto sobre la calidad de la atención. Descriptores: Administración de la seguridad; Certificado de necesidades; Acreditación; Indicadores de calidad de la atención de salud; Contención de riesgos biológicos.
\end{abstract}

\section{INTRODUÇÃO}

A prestação de assistência ao paciente nas instituições hospitalares, nos últimos anos, tem exigido da enfermagem uma atuação extremamente complexa. Neste ínterim, a segurança dos pacientes ganha relevância de "especialidade", evocando a necessidade da enfermagem de se capacitar cientificamente e se comprometer eticamente com ações sistêmicas de avaliação e prevenção, tentando viabilizar a redução de desfechos indesejados, bem como a de analisar o impacto sobre a qualidade do cuidado a eles oferecidos. Considerando a pertinência desta temática, delimitamos para análise, neste ensaio reflexivo, a expressão "práticas seguras no/do cuidado de enfermagem", entendendo tratar-se de discussão multifacetada, com uma diversidade de interesses e de entidades, exigindo, portanto, pensar sobre uma diversidade de aspectos.
Nesse sentido, balizamo-nos em dois pressupostos: primeiro, no pressuposto de que conhecimento algum pode se edificar sobre fundamentos inquestionáveis e sem validação em debates no coletivo social; segundo, no de que a exata medida do erro é complexa. Assim, conhecer e compreender a natureza da ação e dos resultados possíveis de sua execução tornase uma tarefa árdua. Ou seja, nem sempre sabemos como funciona o aparato tecnológico que manejamos.

A adoção destes dois pressupostos nos leva a indagar e responder: como temos nos aliado aos nossos órgãos de classe e a projetos privados e/ou governamentais na tentativa de mudança de uma realidade em busca da melhoria da qualidade e da segurança da assistência prestada aos nossos clientes? Quais são os percursos tentados no sentido de concretizar nossos desejos e assegurar uma assistência que também gostaríamos 
de receber, mas que, antes de tudo, precisamos e temos o dever de oferecer como profissionais comprometidos com os clientes e com a assistência que nos responsabilizamos em fazer? ${ }^{(1)}$ De que modo o cuidar na enfermagem ontem e hoje tem se efetivado? Em termos propositivos, quais recursos técnicos e políticos para ampliação das práticas seguras estão na pauta do dia? Ao considerarmos esta faceta de integração, o que se diz e o que se faz na enfermagem? É disso que tratamos a seguir.

\section{Cuidar na enfermagem ontem e hoje}

$\mathrm{Na}$ contramão de um possível discurso do senso comum que veicularia a noção de as enfermeiras priorizarem a conduta em detrimento do conhecimento técnico, reportamos o estudo de Kruse. ${ }^{(2)}$. Este foi o primeiro a sinalizar as condições da instituição hospitalar da segunda metade do século XIX, com a figura do médico já introjetada, ocupando um espaço privilegiado como detentor de um saber científico otimizado pelas invenções de diversos aparelhos que auxiliam no diagnóstico e tratamento de doenças. Após essa abordagem, a mesma autora analisa a enfermagem em tal contexto de possibilidade, ou seja, o fato de a enfermeira ter um papel determinado na instituição hospitalar daquela época na medida em que o cuidado em si preocupava menos do que a disciplina daqueles que prestavam os cuidados. A disciplina tinha a função de sustentar o próprio hospital como um espaço de cura centrada no tratamento médico. Mesmo assim, a prática da enfermagem toma outro rumo com Florence Nightingale que, decidida a organizar uma profissão, vinculou-a a um saber que tinha status, um saber próximo da ciência ${ }^{(2)}$. Logo, o fato de a enfermeira estar vinculada ao trabalho médico permitiu que ela usufruísse de seu prestígio, enquanto outros cuidadores no hospital não gozavam de um reconhecimento profissional.

E hoje? Banho, higiene oral, alimentação, mudança de decúbito, administração de medicamentos, entre outros cuidados, assumem uma dimensão nunca experimentada pela enfermagem - e nem é preciso fazer referência aos cuidados mais complexos, por exemplo, nas unidades de terapia intensiva. Fiquemos naqueles muitas vezes considerados secundários e questionemos: no contexto hospitalar, é possível ainda considerá-los menores do que o tratamento médico do paciente? Estamos falando, sim, daquele cuidado outrora entendido como insignificante como capaz de interferir diretamente, quando não realizado ou indevidamente operacionalizado, na gravidade dos pacientes, nas complicações e nas sequelas desses mesmos pacientes, no seu tempo de internação hospitalar e até mesmo na sua evolução para o óbito.

A enfermagem hoje fala em gerir seu próprio negócio e no aumento efetivo de profissionais pela sua proximidade do cuidado ao paciente. Mas esse mesmo cuidado tem sido ressignificado política e socialmente, porque também interfere no econômico. É ilusório dizermos que cuidado não gera lucro para a instituição hospitalar; aliás, se indevidamente conduzido, acarreta prejuízo.

Contudo, tem sido disseminada a ideia de que a enfermagem está acoplada a processos mais amplos de produção na saúde. A enfermagem vê seu produto reconhecido, mas como parte de outro, que não raro ofusca sua ação e resultados. O reconhecimento da importância dos componentes de um produto maior depende de suas identificações, pela constatação de que eles existem e requerem conhecimento, habilidades e esforços para serem obtidos ${ }^{(3)}$. Existe, aí, um paradoxo, pois as atuais relações entre paciente e instituições hospitalares sustentam-se na busca de resoluções adequadas por práticas seguras, o que ora ofusca não apenas o processo de trabalho da enfermagem, mas de todos os demais profissionais - inclusive o trabalhador médico - atrelados diretamente no processo do cuidar, ora potencializa toda e qualquer atividade profissional porque capaz de interferir para o bem e para o mal na almejada prática segura. É destacada, pois, em cada profissão envolvida, a sua responsabilidade no cuidar.

Enfim, para atender às transformações tecnológicas, sociais e econômicas, a nossa responsabilidade no cuidar inclui conseguir operar com uma linguagem interdisciplinar - traduzida em indicadores de qualidade, qualidade do cuidado, protocolos assistenciais e/ou do cuidado, gestão de risco, enfermagem baseada em evidências (EBE), eventos adversos, segurança do paciente, acreditação hospitalar, comissão de ética e estratégias preventivas.

\section{Ampliando a discussão das práticas seguras}

Qualidade é relacionada ao nível de satisfação dos usuários dos serviços, supondo que estes recebam assistências oportunas, efetivas e seguras (qualidade técnica dos processos assistenciais) em condições materiais e éticas adequadas (qualidade percebida ou acolhimento) ${ }^{(4)}$. O Ministério da Saúde define qualidade como o grau de atendimento a padrões estabelecidos, frente às normas e protocolos que organizam as ações e práticas, assim como aos atuais conhecimentos técnicos científicos ${ }^{(5)}$.

Já no início do século XX, o médico cirurgião Codman, inspirado em Florence, preconizou a qualidade dos resultados das intervenções médicas nos hospitais. A posteriori, em 1917, o programa de padronização hospitalar realizou auditoria em 692 hospitais americanos, detectando que apenas 89 cumpriam os padrões mínimos estabelecidos na época. 0 Colégio Americano de Cirurgiões aprimorou tais padrões mínimos em 1924(6), refletindo que os padrões seriam acordos sobre a melhor maneira de organizar e executar as ações que conduzem à obtenção dos resultados procurados.

Em 1950 a enfermagem de Washington divulgava os "Princípios científicos aplicados à enfermagem". As 
estudiosas que desenvolveram as teorias de enfermagem, em suas mais diversas perspectivas, foram propulsoras deste debate. No Brasil, em 1977, o Ministério da Saúde sistematizou os padrões mínimos de assistência de enfermagem. Recentemente, intensificaram-se intercâmbios entre mobilizações comprometidas com questões da sistematização da assistência de enfermagem, com os diagnósticos de enfermagem e com os indicadores de processos e de resultados das ações de enfermagem ${ }^{(6)}$.

Paralelamente a estes movimentos, a intitulada Joint Commission on Acreditation of Hospitals (JCAHO) é instituída em 1959 e alterada em 1987 para Comissão Conjunta de Acreditação de Organizações de Assistência à Saúde ${ }^{(6)}$. Atualmente, a Joint Commission International $(\mathrm{JCI})^{(7)}$, ao definir segurança como ausência de exposição ao perigo e proteção contra ocorrência ou risco de lesão ou perda, estabeleceu diretrizes e metas internacionais de segurança para os pacientes que acessam os serviços hospitalares. A JCl, em 2007, colocou em teste piloto as avaliações e manutenção de acreditação dos hospitais por meio das Metas Internacionais de Segurança do Paciente. Estas metas são avaliadas e pontuadas com os padrões conforme (C), parcialmente conforme (PC) e não conforme (NC). Cada meta possui elementos de mensuração específicos que são avaliados isoladamente em relação aos padrões, e incluem: identificação correta dos pacientes; melhoria na efetividade da comunicação entre os profissionais da assistência; aperfeiçoamento da segurança no uso de medicações de alto risco; eliminação de cirurgias em lado-errado, paciente-errado, procedimentos-errados; redução dos riscos de infecção; redução dos riscos de dano/lesão ao paciente vítima de queda.

A implantação do processo pela $\mathrm{JCl}^{(7)}$ criou normas e mecanismos de avaliação para o controle da qualidade assistencial pelos profissionais de saúde. Entre as atividades recomendadas aos hospitais para a segurança do paciente, estão: estabelecimento de rotina de coleta e análise de dados relacionados à segurança; registro e notificação de incidentes e de erro de medicação; vigilância de infecção e da segurança das instalações; percepções da equipe e sugestões para melhoria da segurança do paciente; espontaneidade da equipe em notificar erros; percepções e sugestões do paciente/família para melhoria da segurança do paciente; identificação e registro de eventos sentinela; redução proativa de riscos; identificação de processos de alto risco e análise crítica de falhas; divulgação dos resultados para o programa de segurança do paciente, para a equipe da organização, para a liderança executiva e para a direção do hospital.

Na ampliação da discussão das práticas seguras, destacamos a Organização Nacional de Acreditação $(\mathrm{ONA})^{(8)}$, ligada ao Ministério da Saúde. Os serviços hospitalares em avaliação pela ONA englobam liderança e administração; serviços profissionais e organização da assistência - em que a enfermagem está inserida; serviços de atenção ao paciente/ cliente; serviço de apoio ao diagnóstico; serviços assistenciais e de abastecimento; serviços de infraestrutura e apoio logístico; ensino e pesquisa. Para fins de avaliação, a ONA distingue três níveis. Para atingir o nível 1, as instituições necessitam priorizar a estrutura e a segurança; no nível 2, acrescenta-se a descrição e o registro dos processos internos, e, para a melhor acreditação (nível 3), devem enfocar os processos vinculados à sua estratégia e melhoria contínua da qualidade. Contudo, as instituições não atingem, normalmente, o nivel 3 de acreditação por não utilizarem indicadores epidemiológicos no planejamento e na definição do modelo assistencial e na comparação de resultados obtidos com referenciais, nem na análise do impacto gerado junto à comunidade e ao sistema de análise da satisfação dos usuários ${ }^{(8)}$.

A Associação de Enfermeiros dos Estados Unidos (ANA), em 1995, recomendou alguns indicadores de qualidade focados no paciente: infecção hospitalar; taxa de dano ao paciente; manutenção da integridade da pele; total de horas de enfermagem fornecidas ao paciente dia (ajustado à gravidade); satisfação do paciente com cuidado de enfermagem, com o gerenciamento da dor e com a educação para saúde recebida; avaliação e implementação dos cuidados requeridos pelo paciente. A ANA busca avaliar também a satisfação da própria equipe de enfermagem. Outra tentativa é vinculada ao sistema de Classificação de Resultados do Paciente (NOC), desenvolvido em 1997 pelo mesmo grupo responsável pela Classificação de Intervenções de Enfermagem (NIC), sendo que a NOC é considerada como uma classificação integral e normalizada dos resultados obtidos com os pacientes, desenvolvida para avaliar os efeitos das intervenções de enfermagem ${ }^{(9)}$.

Na questão do gerenciamento de riscos, existe alinhamento entre a ANA, a JCl e o "Institute for Healthcare Improvement" (IHI). O IHI ${ }^{(10)}$ tornou-se conhecido por lançar nos Estados Unidos uma campanha de proteção a cinco milhões de vidas, elegendo alguns problemas para desencadear uma resposta proativa por meio do time de resposta rápida, da redução de complicações cirúrgicas ou decorrentes de cirurgia, da identificação do paciente, do gerenciamento da dor e do desenvolvimento de protocolos que pudessem desencadear prevenção de mortes relacionadas ao infarto agudo do miocárdio (IAM), de eventos adversos com medicamentos, de infecção relacionada ao uso de recursos, de infecções de sítio cirúrgico, de pneumonia associada à ventilação mecânica (PAV), da trombose venosa profunda, da hemorragia digestiva, de úlcera de pressão (UP), de infecção por Staphylococcus Aureus Methicillin-Resistente (MRSA), de danos ou injúrias relacionados a eventos adversos. No Brasil, 
muitas instituições hospitalares lançaram sua plataforma de prevenção centrando-se no IAM, nos eventos adversos por medicamentos, na infecção por cateter venoso central, na UP, na infecção em sítios cirúrgicos, no MRSA e no monitoramento da PAV.

A iniciativa mais recente de atualizações acerca das pesquisas que envolvem a prática da enfermagem é a Evidence-based Nursing ${ }^{(9)}$. A origem da Enfermagem Baseada em Evidências (EBE) está no movimento da medicina com base em evidências, ou seja, nesse contexto de urgente adoção de medidas que minimizem o distanciamento entre os avanços científicos e a prática assistencial. A EBE desconsidera a experiência clínica isolada e não-sistemática, as opiniões infundadas e a tradição como base para a prática de enfermagem. Ela reforça a utilização de resultados de pesquisas e, na ausência desses, de dados obtidos de forma sistemática, oriundos de programas de avaliação e de melhoria de qualidade e/ou consenso de especialistas reconhecidos e de confirmada experiência para comprovar a prática. A EBE é um processo que consiste em cinco etapas: a formulação de questões originárias da prática profissional; a investigação da literatura ou outros recursos relevantes de informações na busca das evidências; a avaliação das evidências em relação à validade, generalização e transferência; de conhecimento do uso da melhor evidência disponível, habilidade clínica e as preferências do cliente no planejamento e implantação do cuidado e a avaliação do enfermeiro em relação à sua própria prática. Além disso, é apreendido que questões de pesquisa orientadas para a causa, prognóstico, diagnóstico, prevenção, tratamentos ou custos sobre problemas de saúde são mais bem respondidas utilizandose a abordagem quantitativa; enquanto que questões sobre o significado da experiência de doença e compreensão dos sentimentos do paciente sobre os efeitos de uma intervenção, com a abordagem qualitativa ${ }^{(11)}$.

Entretanto, no Brasil, várias são as barreiras que dificultam a utilização de resultados de pesquisas, tais como a falta de preparo da enfermeira, o fato de essa profissional não perceber a pesquisa como parte integrante do seu cotidiano profissional, falta de tempoe suporte organizacional (recursos humanos, materiais e financeiros). Mesmo assim, compete à enfermeira buscar, na literatura, revisões sistemáticas já elaboradas, as quais respondam a questionamentos sobre o melhor cuidado a ser prestado, bem como realizar uma avaliação crítica da sua aplicação ao seu cotidiano ${ }^{(12)}$.

O Processo de Enfermagem (PE), para ser efetivo, exige uma preocupação holística com o paciente e a identificação da necessidade de cuidados balizada em uma prática que incorpora resultados de pesquisa ao cuidado diário, realizado nas instituições de saúde. Logo, há necessidade de estudos com metodologias que fundamentem um cuidado de enfermagem seguro e qualificado, favorecendo-lhe uma posição de destaque no âmbito da interdisciplinaridade que lida com o paciente e familiar, seja na assistência ou na gestão. Neste caso, os protocolos de cuidados, com base no princípio da EBE, constituem-se em mais uma ferramenta que pode complementar com qualidade os PE e nortear a construção de sistemas de avaliação de eficiência e eficácia. Outras motivações para a utilização de protocolos clínicos, incluem a proteção contra o risco de acusações relativas à prática profissional e a redução de custos. Todavia, pela velocidade com que novas tecnologias de cuidado são introduzidas na saúde, a enfermagem deve fazer revisão anual, ou no máximo a cada dois anos ${ }^{(9)}$.

Ainda, como ferramenta de avaliação da qualidade do cuidado, tem sido preconizada a utilização de indicadores. Um indicador pode ser definido como uma unidade de medida de uma atividade, embora não seja uma medida direta de qualidade. Os indicadores sinalizam divergências com o padrão desejável e atuam como uma chamada identificando e direcionando a atenção para os pontos-chave do cuidado que necessitam ser revistos. Para isso, os indicadores devem ser os mais específicos possíveis na questão tratada, sensíveis a mudanças específicas nas condições de interesse $\mathrm{e}^{(5)}$.

Frente à necessidade de garantir a qualidade do cuidado, com menores riscos ao paciente, torna-se premente articular as práticas seguras a uma discussão redimensionada a partir do conceito de evento adverso. Evento adverso é tema preocupante que está em pauta por se encontrar visível e grosseiramente avolumado. Trata-se de evento que ocorre durante a assistência à saúde e que pode causar uma injúria, dano físico ou psicológico ao paciente ou à organização. $O$ evento adverso grave pode resultar em morte, risco de vida ou prolongamento da hospitalização. Por sua vez, o evento sentinela é aquele que, embora com potencial de dano presente, não resulta em dano real. Já a quase falha é um evento em que as consequências indesejáveis foram revertidas porque a descoberta com antecedência possibilita sua correção(13).

Nesta perspectiva, com as novas exigências de segurança, muitos hospitais reconhecem a necessidade de desenvolver estratégias preventivas que impeçam ou minimizem a chance de erros e falhas em seus serviços tornando, o processo hospitalar mais seguro e confiável aos pacientes e à população. Em algumas instituições, essa preocupação tem se viabilizado através do Serviço de Epidemiologia e Gestão de Risco, que é o sistema de notificação de situações iatrogênicas que ocorrem com os pacientes ${ }^{(13)}$. Outras instituições sustentam este monitoramento a partir dos Comitês de Ética - entre estes, o Comitê de Ética em Enfermagem. A ideia é a de não identificar os profissionais culpados, mas descobrir lacunas nos processos a serem revistos, em benefício do paciente e de toda a equipe envolvida.

Mas como fazer isso? É imprescindível que os gerentes dos serviços de enfermagem saibam requerer das instituições a responsabilidade administrativa, ética e legal de dimensionar o quadro funcional, investir em recursos humanos e materiais ${ }^{(14)}$. Além disso, a prática educativa é de fundamental importância 
na enfermagem, tanto para o paciente quanto para o profissional. Assim, a instituição tem a responsabilidade de propor programas apropriados às necessidades de qualificação em novas situações e reciclar seus trabalhadores, qualificandoos para as exigências assistenciais ou para as mudanças necessárias frente à assistência e qualidade.

\section{Quando dizer e fazer são faces da mesma moeda}

Na seção anterior, mesmo sem a pretensão de esgotar o assunto, mapeamos algumas perspectivas de discussão, na atualidade, acerca da temática "práticas seguras". De algumas perspectivas, sobressai um caráter mais científico; de outras, acentua-se o aspecto legal e bioético; de mais algumas, um delineamento mais econômico; de todas elas, advém o político, porque inseridas em debates coletivos e em mecanismos de interesses. Práticas que reforçam a máxima "constituir-se em sujeito de conduta e, ao mesmo tempo, sujeito do conhecimento", que busca a instrumentalização - não só técnica, mas a política, para a compreensão do contexto em que se vive e de possíveis intervenções a serem feitas.

Essas considerações são importantes para pensar o trabalho na enfermagem, pois entendemos que onde parece nada existir além de regra e mais disciplina, é justamente onde pode residir o maior potencial de resistência e recriação. Ressignificar o cuidar em enfermagem, ampliar a discussão das práticas seguras no/do cuidado de enfermagem, levou-nos a refletir acerca do nosso potencial de realização.

Estima-se que bem mais de $50 \%$ das atuais ações propostas para a efetivação de práticas seguras passa pelo cuidar em enfermagem. Estudos publicados ou apresentados em eventos científicos, evidenciam que a elaboração e a aplicação de protocolos do cuidado ou assistenciais impactam sobre a segurança e a qualidade do cuidado, atrelados, entre outros aspectos, à aceleração da recuperação, à redução de uma ou mais complicações, falhas e eventos adversos, à redução do tempo de internação e do custo hospitalar, à organização da equipe de trabalho e à satisfação dos pacientes e familiares.

Como exemplos, reportamo-nos às iniciativas da enfermagem na implementação de protocolos de cuidado para fornecimento de informações e orientações; pré e pós-operatório cirúrgico; transplante de órgãos; monitorização hemodinâmica; manejo não-farmacológico da dor e prevenção da PAV; tratamento de feridas e prevenção do desenvolvimento de úlcera de pressão; utilização de protocolos de insulina; redução da necessidade de internação hospitalar de pacientes com insuficiência cardíaca.

Escolher a melhor prática de aspiração de secreções das vias aéreas inferiores e avaliar o padrão de segurança deste procedimento na interface com a infecção e com as alterações hemodinâmicas constituem-se em um dos exemplos para minimizar os possíveis efeitos danosos do procedimento em si ao paciente. Outro viés de cuidado direciona para os erros com medicações. Contudo, estamos na fase de detectar os erros e os eventos adversos para estabelecer medidas estruturais e educativas no combate a este tipo de situação.

Após sinalizar alguns exemplos em que dizer e fazer são faces da mesma moeda, finalizamos argumentando que é preciso incorporar o debate entre os profissionais, refletir acerca dos valores envolvidos nas situações e adotar uma abordagem de precaução priorizando tanto os resultados, quanto os processos avaliativos das ações propostas. O pressuposto é compreender que estamos sempre na possibilidade de incorrer no erro de desenvolvermos ações que não resultam no aprimoramento do processo de segurança e da qualidade do cuidado ao paciente.

\section{Referências}

1. Lunardi VL. Bioética aplicada à assistência de enfermagem. Rev Bras Enferm.

1998;51(4):655-64.

2. Kruse MHL. Os poderes dos corpos frios. Das coisas que se ensinam às

enfermeiras. Brasilia: ABEn; 2004.

3. Erdmann AL, Silva MA, Erdmann RH, Ribeiro JA. O reconhecimento do produto

do sistema organizacional de cuidados de enfermagem. Ciênc Cuid e Saúde.

2005;4(1):37-46

4. Organização Pan-Americana de Saúde. A gestão da qualidade.

In: Organização Pan-Americana de Saúde. A transformação da gestão de

hospitais na América Latina e Caribe. Brasília: OPAS/OMS; 2004. p. 215-49.

5. Vituri DW, Matsuda LM. Validação de conteúdo de indicadores

de qualidade para avaliação do cuidado de enfermagem.

Rev Esc Enferm USP. 2009;43(2):429-37.

6. Feldman LB. Como alcançar a qualidade nas instituiçōes de saúde.

Critérios de avaliações, procedimentos de controle, gerenciamento de riscos

hospitalares até a certificação. São Paulo: Martinari; 2004.

7. Consórcio Brasileiro de Acreditação de Sistemas e Serviços de Saúde.
Padrōes de acreditação da Joint Comission International para hospitais. $3^{\mathrm{a}}$ ed.

Rio de Janeiro: CBA; 2008.

8. Organização Nacional de Acreditação. Diretrizes do sistema e do processo de

acreditação. Brasília: ONA; 2007.

9. Jung G. O estado da arte: enfermagem baseada em evidências

e os protocolos clínicos. In: Mancia JR, Vargas MAO. Livro-Tema da

66a Semana Brasileira de Enfermagem. A bioética e os modos de ser da

enfermagem. Brasilia: ABEn; 2005. p. 58-74.

10. Institute for Healthcare Improvement. Protecting 5 million lives [Internet].

[citado em 2009 Ago 07]. Disponível em: http://www.ihi.org/compaign

11. Galvão MC, Sawada NO, Mendes IA. A busca das melhores evidências.

Rev Esc Enferm USP. 2003;37(4):43-50.

12. Galvão MC, Sawada NO, Trevisan MA. Revisão sistemática: recurso que

proporciona a incorporação das evidências na prática da enfermagem.

Rev Latino-am Enferm. 2004;12(3):249-56.

13. Berquó L. Serviço de epidemiologia e gestão de risco.

Porto Alegre: Hospital Mãe de Deus; 2007. 